\title{
NON-UNIFORM FILTER BANKS BASED ON A MULTI-PROTOTYPE COSINE MODULATION
}

\author{
F.Argenti and E.Del Re \\ Dipartimento di Ingegneria Elettronica, Universitá di Firenze \\ Via di Santa Marta, 3 - 50139 Firenze - Italy \\ argenti@cosimo.ing.unifi.it
}

\begin{abstract}
In this work a method to design non-uniform FIR filter banks is presented. It is based on the cosine modulation of more than one prototype, having different passbands. The method aims at the cancellation of the main component of aliasing: this imposes constraints on the prototypes, which become dependent of each other. The method is simple and requires numerical optimization only for the narrowest prototype, being the others derived in a straightforward way from this one. For this reason, the method seems particularly suitable when banks with a large number of coefficients must be designed.
\end{abstract}

\section{INTRODUCTION}

In some applications a non-uniform splitting of the input signal spectrum could be preferable to a uniform one. An example is perceptual compression of audio signals, where non-uniform frequency intervals match better the critical bands of the human auditory system [1][2].

Non-uniform filter banks have been analyzed, for example, in [3]-[7]. In [4] the concept of compatible sets is introduced: a necessary condition that allows aliasing cancellation is that the decimation factors of the banks form a compatible set. In [5] analysis and design methods for nonuniform banks are carried out in the time domain. In [6] two transforms that permit to represent banks with rational sampling factors through uniform banks are introduced and design methods that allow perfect reconstruction are presented. In [7] a design method based on the cancelling of the main component of aliasing is shown. It is also applied in [8] to the design of Short-Time Fourier transformers. The method that is proposed here is valid for integer decimation factors and, as that shown in [7], is based on the cosine modulation of different linear phase prototypes having a proper passband. A first prototype, having the narrowest passband, is designed by using lattice structures [9]. The cancellation of the main aliasing component imposes constraints on the other prototypes: this leads to their specification in the frequency domain and permits the computation of their impulse responses.

\section{MULTI-PROTOTYPE COSINE MODULATED BANKS}

Consider the system in Fig. 1, where a non-uniform bank having integer decimation factors $M_{k}, \mathrm{k}=0, \ldots, \mathrm{K}-1$, is depicted. The input-output relationship in the z-domain is given by:

$$
\hat{X}(z)=\sum_{k=0}^{K-1} \frac{1}{M_{k}} \sum_{l=0}^{M_{k}} H_{k}\left(z W_{M_{k}}^{l}\right) X\left(z W_{M_{k}}^{l}\right) F_{k}(z)
$$

where $W_{M}=e^{-j 2 \pi / M}$. In the synthesis stage, the filter $F_{k}(z)$ extracts the term $H_{k}(z) X(z)$, obtained for $1=0$, but aliasing components, given by the terms in the summation relative to $l \neq 0$, are also produced.

We will suppose that the filters of the bank are obtained through cosine modulation of a small number of prototypes, having different passbands. Cosine modulation can be used to design Perfect Reconstruction [9] and Pseudo-QMF [10] uniform banks.

For simplicity's sake consider the case of two real linear phase prototypes, $h(n)$ and $g(n)$, that permit to build two uniform banks, with $M_{1}$ and $M_{2}$ channels, respectively. A non-uniform bank will be achieved by selecting filters from the two banks so that the input spectrum is completely covered. The passband widths and their positions in the spectrum must satisfy proper conditions [6]. The filter impulse responses are given by:

$$
\begin{aligned}
\operatorname{Bank1} & \\
h_{1, k}(n) & =2 h(n) \cos \left((2 k+1) \frac{\pi}{2 M_{1}}\left(n-\frac{N_{1}-1}{2}\right)+\theta_{1, k}\right) \\
f_{1, k}(n) & =2 h(n) \cos \left((2 k+1) \frac{\pi}{2 M_{1}}\left(n-\frac{N_{1}-1}{2}\right)-\theta_{1, k}\right)= \\
& =h_{1, k}\left(N_{1}-1-n\right)
\end{aligned}
$$

for $k=0,1, \ldots, M_{1}-1$ and by

$$
\begin{aligned}
\operatorname{Bank2} & \\
h_{2, m}(n) & =2 g(n) \cos \left((2 m+1) \frac{\pi}{2 M_{2}}\left(n-\frac{N_{2}-1}{2}\right)+\theta_{2, m}\right) \\
f_{2, m}(n) & =2 g(n) \cos \left((2 m+1) \frac{\pi}{2 M_{2}}\left(n-\frac{N_{2}-1}{2}\right)-\theta_{2, m}\right)= \\
& =h_{2, k}\left(N_{2}-1-n\right)
\end{aligned}
$$

for $m=0,1, \ldots, M_{2}-1 . \quad N_{1}$ and $N_{2}$ are the length of the two prototypes. The prototypes are linear phase and satisfy $h(n)=h\left(N_{1}-1-n\right)$ and $g(n)=g\left(N_{2}-1-n\right)$. The 


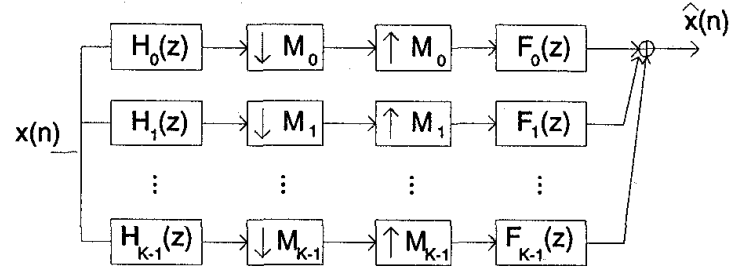

Figure 1: Non-uniform filter banks having integer decimation factors.

two phase terms $\theta_{1, k}$ and $\theta_{2, m}$ are introduced to eliminate aliasing components.

The prototypes $h(n)$ and $g(n)$ must be designed so that the main components of aliasing in (1), coming from adjacent filters, are eliminated. Consider the frequency responses of Bank 1 analysis filters:

$$
H_{1, k}(F)=H_{1, k}^{l o w}(F)+H_{1, k}^{u p}(F)
$$

where

$$
\begin{array}{r}
H_{1, k}^{l o w}=e^{j\left[(2 k+1) \frac{\pi}{2 M_{1}} \frac{N_{1}-1}{2}-\theta_{1, k}\right]} H\left(F+\frac{2 k+1}{4 M_{1}}\right) \\
H_{1, k}^{u p}(F)=e^{j\left[-(2 k+1) \frac{\pi}{2 M_{1}} \frac{N_{1}-1}{2}+\theta_{1, k}\right]} H\left(F-\frac{2 k+1}{4 M_{1}}\right)
\end{array}
$$

and where $H(F)$ is the Fourier Transform of $\mathrm{h}(\mathrm{n})$. Eq. (4) shows that the frequency response of $H_{1, k}(z)$ is composed by a right-shifted and a left-shifted version of $H(F)$. Similar definitions hold for $F_{1, k}(z)$ and for Bank 2 .

Suppose now that the k-th filter of Bank 1 and the mth filter of Bank 2 have adjacent bandwidths: suppose that the latter covers on the positive frequency axis higher frequencies than the first. Here following, some facts known from uniform pseudo-QMF bank theory (see, for example, [3][11]), that hold also for non-uniform banks, are reviewed.

Consider the $\mathrm{k}$-th synthesis filter of Bank 1 and let $A_{1, u p}$ be the aliasing term at the high frequency edge on the positive frequency axis, which is the product of $F_{1, k}^{u p}(F)$ and $H_{1, k}^{l o w}\left(F-\frac{k+1}{M_{1}}\right)$, i.e.,

$$
\begin{aligned}
A_{1, u p}= & \frac{1}{M_{1}} e^{-j 2 \theta_{1, k}} H\left(F-\frac{2 k+1}{4 M_{1}}\right) H\left(F-\frac{2 k+3}{4 M_{1}}\right) . \\
& \cdot X\left(F-\frac{k+1}{M_{1}}\right)
\end{aligned}
$$

Consider also the aliasing components produced in Bank 2 and let $A_{2, \text { low }}$ be the aliasing term at the low frequency edge of $F_{2, m}^{u p}(F)$, given by the product of $F_{2, m}^{u p}(F)$ and $H_{2, m}^{\text {low }}\left(F-\frac{m}{M_{2}}\right)$, i.e.,

$$
\begin{aligned}
A_{2, \text { low }}= & \frac{1}{M_{2}} e^{-j 2 \theta_{2, m}} G\left(F-\frac{2 m+1}{4 M_{2}}\right) G\left(F-\frac{2 m-1}{4 M_{2}}\right) . \\
& \cdot X\left(F-\frac{m}{M_{2}}\right)
\end{aligned}
$$

The main component of aliasing is eliminated if

$$
A_{1, \text { up }}+A_{2, \text { low }}=0
$$

which imposes a condition on the indexes $\mathrm{k}$ and $\mathrm{m}$, i.e.,

$$
\frac{k+1}{M_{1}}=\frac{m}{M_{2}}
$$

Moreover, if we impose the following condition

$$
\begin{aligned}
& \frac{1}{M_{1}} H\left(F-\frac{2 k+1}{4 M_{1}}\right) H\left(F-\frac{2 k+3}{4 M_{1}}\right) \\
& =\frac{1}{M_{2}} G\left(F-\frac{2 m+1}{4 M_{2}}\right) G\left(F-\frac{2 m-1}{4 M_{2}}\right)
\end{aligned}=
$$

then eq. (9) yields

$$
e^{-j 2 \theta_{1, k}}+e^{-j 2 \theta_{2, m}}=0
$$

that shows the well-known result that the phase terms relative to adjacent filters must differ of $\pi / 2$.

Consider now the zero-phase filters associated with each linear phase prototype defined as

$$
\begin{aligned}
& H_{0}(F)=H(F) e^{j 2 \pi F \frac{N_{1}-1}{2}} \\
& G_{0}(F)=G(F) e^{j 2 \pi F \frac{N_{2}-1}{2}}
\end{aligned}
$$

If the prototypes have the same length, i.e., $N_{1}=N_{2}$, then (11) becomes

$$
\begin{aligned}
& \frac{1}{M_{1}} H_{0}\left(F-\frac{2 k+1}{4 M_{1}}\right) H_{0}\left(F-\frac{2 k+3}{4 M_{1}}\right) \\
& =\frac{1}{M_{2}} G_{0}\left(F-\frac{2 m+1}{4 M_{2}}\right) G_{0}\left(F-\frac{2 m-1}{4 M_{2}}\right)
\end{aligned}=
$$

Therefore, the prototypes can not be designed independently of each other. Eq. (14) is satisfied if the frequency responses of the filters $H_{0}(F)$ and $G_{0}(F)$ satisfy the following two conditions: 1) they have an infinite attenuation in stopband and 2) the magnitudes are proportional to each other in the transition band.

Once the main aliasing component has been approximately eliminated, the input-output relationship becomes

$$
\hat{X}(z) \cong \sum_{k=0}^{K-1} \frac{1}{M_{k}} H_{k}(z) F_{k}(z) X(z)=T(z) X(z)
$$

Therefore, in the absence of aliasing, perfect reconstruction could be achieved if $T(z)$ is allpass, i.e.,

$$
|T(F)|=\sum_{k=0}^{K-1} \frac{1}{M_{k}}\left|H_{k}(F)\right|^{2}=1
$$

A procedure to design the prototypes is now described. It is assumed that $\mathrm{H}(\mathrm{F})$ has a passband narrower than $\mathrm{G}(\mathrm{F})$ and it is supposed that the values $M_{1}, M_{2}$ and $N=N_{1}=$ $N_{2}$ are given.

1) Design a linear phase filter $\mathrm{H}(\mathrm{F})$ with cut-off frequency $F_{t}=\frac{1}{4 M_{1}}$ and characterized by a power complementary transition band, i.e.,

$$
|H(F)|^{2}+\left|H\left(F-\frac{1}{2 M_{1}}\right)\right|^{2}=1
$$


for $0<F<\frac{1}{2 M_{1}}$. We have used the method described in [9], that permits to design prototypes satisfying (17) by using lattice structures. One of the advantages of this method is that the lattice parameters are obtained by minimizing an objective function that considers only the behavior of the filter in the stopband. In this case, the length of the prototype must satisfy $N=2 m M_{1}$, with $\mathrm{m}$ an integer.

2) Find the zero-phase filter $H_{0}(F)$ related to $\mathrm{H}(\mathrm{F})$. $H_{0}(F)$ is a real function symmetrical around $\mathrm{F}=0$.

3) Build the zero-phase frequency response $G_{0}(F)$. Let $F_{t}=\frac{1}{4 M_{2}}-\frac{1}{4 M_{1}}$ and let $G_{0}(F)$, for $0<F<0.5$, be:

$$
G_{0}(F)= \begin{cases}\sqrt{\frac{M_{2}}{M_{1}}} & 0<F<F_{t} \\ \sqrt{\frac{M_{2}}{M_{1}}} H_{0}\left(F-F_{t}\right) & F_{t}<F<0.5\end{cases}
$$

Moreover $G_{0}(F)$ is symmetrical around $\mathrm{F}=0$. As a result of this definition, the prototype $G_{0}(F)$ is obtained by dropping information in the stopband of $H_{0}(F)$, while a part of the passband is taken equal to the ideal value.

4) Use (18) and (13) to achieve $G(F)$ and, therefore, $\mathrm{g}(\mathrm{n})$. This can be accomplished in different ways. By using the frequency sampling method, $g(n)$ is computed as the IDFT of $\mathrm{N}$ uniformly spaced samples of $G(F)$. By using window based methods, $g(n)$ is computed as the inverse Fourier Transform of $G(F)$ windowed by an $\mathrm{N}$-length sequence $w(n)$. The experimental results that will be shown in the next section have been obtained with the frequency sampling method.

5) Build the two uniform banks by cosine modulation: selecting filters from each bank so that the spectrum is completely covered and using the correct phase terms that satisfy (12) yields the non-uniform bank.

The procedure that has been described can be applied also when more than two prototypes need to be designed: in this case, lattice structures are used to design the prototype having the narrowest bandwidth, while steps 3) and 4) are repeated for each of the other prototypes.

The effectiveness of the method that is proposed has been tested and the results are shown in the following section.

\section{EXPERIMENTAL RESULTS}

We have considered two examples of non-uniform banks:

Example 1: non-uniform bank composed by three channels and decimation factors $M_{0}=2, M_{1}=6$ and $M_{2}=3$.

Example 2: non-uniform bank composed by five channels and decimation factors $M_{0}=4, M_{1}=12, M_{2}=M_{3}=$ 6 and $M_{4}=3$. This example has been also considered in [7].

Therefore, three and four prototypes must be designed in Example 1 and 2, respectively: the narrowest prototype is designed as described in [9], the others with the procedure described above. The results are evaluated by considering both the transfer function $T(F)$ in the absence of aliasing, given by (16), and the aliasing error. As to the latter measure, a frequency domain estimation of the aliasing error is shown in [9] for the uniform case. Here, the same measure is applied to the non-uniform case as follows. Let $\mathrm{L}$ be the least common multiple of the $M_{k}$ 's and $r_{k}, k=0, \ldots, K-1$, be integers such that $L=M_{k} r_{k}$. By using the transformation described in [4], the non-uniform bank can be represented through a L-channel uniform bank. The k-th analysis/synthesis branch of the non-uniform bank is substituted by $r_{k}$ new branches having $\mathrm{L}$ as decimation/interpolation factor, $z^{p M_{k}} H_{k}(z), p=0, \ldots, r_{k}-1$ as analysis filter and $z^{-p M_{k}} F_{k}(z), p=0, \ldots, r_{k}-1$ as synthesis filter. Therefore, the reconstructed signal is given by

$$
\hat{X}(z)=\sum_{l=0}^{L-1} X\left(z W_{L}^{l}\right) A_{l}(z)
$$

where

$$
A_{l}(z)=\frac{1}{L} \sum_{k=0}^{K-1} H_{k}\left(z W_{L}^{l}\right) F_{k}(z) \sum_{p:=0}^{r_{k}-1} W_{L}^{l p M_{k}}
$$

The last summation in (20) is equal to $r_{k}$ when $l$ is a multiple of $r_{k}$ and zero elsewhere. The aliasing components are given by $A_{l}$ when $l \neq 0$, while the global aliasing error is computed as

$$
E_{a}(F)=\left[\sum_{l=1}^{L-1}\left|A_{l}\left(e^{j 2 \pi F}\right)\right|^{2}\right]^{1 / 2}
$$

The design of the bank of Example 1 has been carried out assuming different lengths of the prototypes, i.e., $\mathrm{N}=\mathbf{2 4}$, 48 and 96 . Table I summarizes the results and reports: the difference between the gains at $\mathrm{F}=0$ and at the first ripple in the stopband for the narrowest prototype; the peak-topeak error $\left(E_{p-p}\right)$ of $|T(F)|$; the maximum value of $E_{a}(F)$. Table II summarizes similar results relative to Example 2. The frequency responses of the filters of: the final analysis bank obtained for Example 2 with $\mathrm{N}=48$ are shown in Fig. 2 , while the transfer function $|T(F)|$ and the aliasing error $E_{a}(F)$ are shown in Fig. 3 and Fig. 4, respectively.

Table I

Results relative to Example 1

\begin{tabular}{|c|c|c|c|}
\hline $\mathrm{N}$ & $\begin{array}{c}\text { Stopband gain } \\
\text { (first ripple) }\end{array}$ & $E_{p-p}$ & $\max E_{a}(F)$ \\
\hline 24 & $-30.6 \mathrm{~dB}$ & $1.65 \mathrm{E}-02$ & $4.69 \mathrm{E}-02$ \\
\hline 48 & $-43.2 \mathrm{~dB}$ & $3.02 \mathrm{E}-03$ & $1.21 \mathrm{E}-02$ \\
\hline 96 & $-51.7 \mathrm{~dB}$ & $2.97 \mathrm{E}-05$ & $2.11 \mathrm{E}-03$ \\
\hline
\end{tabular}

Table II

Results relative to Example 2

\begin{tabular}{|c|c|c|c|}
\hline $\mathrm{N}$ & $\begin{array}{c}\text { Stopband gain } \\
\text { (first ripple) }\end{array}$ & $E_{p-p}$ & $\max E_{a}(F)$ \\
\hline 48 & $-29.8 \mathrm{~dB}$ & $1.53 \mathrm{E}-02$ & $4.53 \mathrm{E}-02$ \\
\hline 96 & $-41.7 \mathrm{~dB}$ & $3.24 \mathrm{E}-03$ & $1.49 \mathrm{E}-02$ \\
\hline
\end{tabular}




\section{CONCLUSIONS}

In this work a new method to design non-uniform filter banks has been presented. It is based on the cosine modulation of more than one prototype. A procedure to design the prototypes has been described and some experimental results have been carried out to show the effectiveness of the design method.

\section{REFERENCES}

[1] N. Jayant, J. Johnston and R.Safranek, "Signal Compression Based on Models of Human Perception", Proceedings of the IEEE, Vol. 81, no. 10, pp. 1385-1422, Oct. 1993.

[2] P. Noll, "Wideband Speech and Audio Coding", IEEE Commun. Mag., pp. 34-44, Nov. 1993.

[3] R.V. Cox, "The Design of Uniformly and Nonuniformly Spaced Pseudoquadrature Mirror Filters", IEEE Trans. Acoust., Speech, Signal Processing, Vol. ASSP-34, no. 5, pp. 1090-1096, Oct. 1986.

[4] P.Q. Hoang and P.P. Vaidyanathan, "Non-uniform Multirate Filter Banks: Theory and Design", in Proc. Int. Symp. Circuits Syst., pp. 371-374, May 1986.

[5] K. Nayebi, T.P. Barnwell III and M.J.T. Smith, "Nonuniform Filter Banks: A Reconstruction and Design Theory", IEEE Trans. Signal Processing, Vol. 41, no. 3, pp. 1114-1127, Mar. 1993.

[6] J. Kovacevic and M. Vetterli, "Perfect Reconstruction Filter Banks with Rational Sampling Factors", IEEE Trans. Signal Processing, Vol. 41, no. 6, pp. 2047-2066, Jun. 1993.

[7] S. Wada, "Design of Nonuniform Division Multirate FIR Filter Banks", IEEE Trans. Circuits Syst. II, Vol. 42, no. 2, pp. 115-121, Feb. 1995.

[8] S.Wada, "Generalized Short-Time Fourier Transforms Based on Nonuniform Filter Bank Structure", Trans. IEICE, Vol. E78-A, no. 3, pp. 431-436, March 1995.

[9] R.D Koilpillai and P.P. Vaidyanathan, "CosineModulated FIR Filter Banks Satisfying Perfect Reconstruction", IEEE Trans. Signal Processing, Vol. 40, no. 4, pp. 770-783, Apr. 1992.

[10] R.D Koilpillai and P.P. Vaidyanathan, "A Spectral Factorization Approach to Pseudo-QMF Design", IEEE Trans. Signal Processing, Vol. 41, no. 1, pp. 8292, Jan. 1993.

[11] P.L. Chu, "Quadrature Mirror Filter Design for an Arbitrary Number of Equal Bandwidth Channels", IEEE Trans. Acoust., Speech, Signal Processing, Vol. ASSP33, no. 1, pp. 203-218, Feb. 1985.

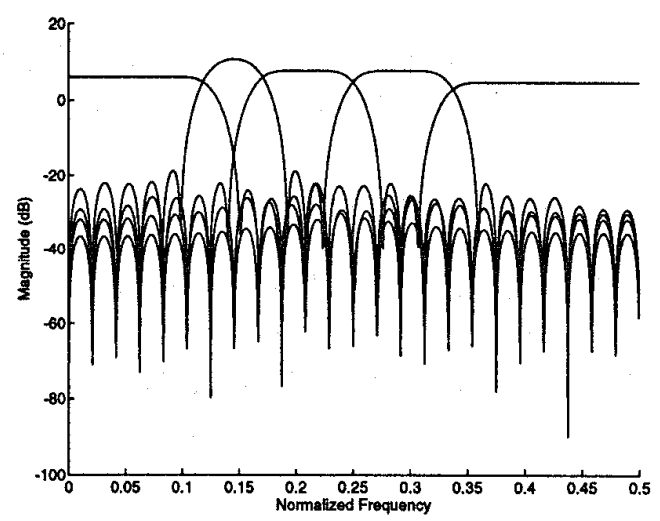

Figure 2: Frequency responses of the analysis filters of the bank of Example $2(\mathrm{~N}=48)$

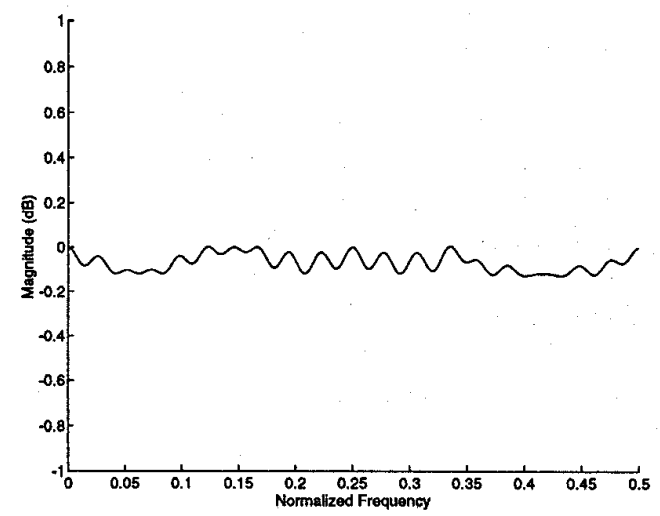

Figure 3: Overall transfer function $\left(20 \log _{10}|T(F)|\right)$ for the bank of Example $2(\mathrm{~N}=48)$

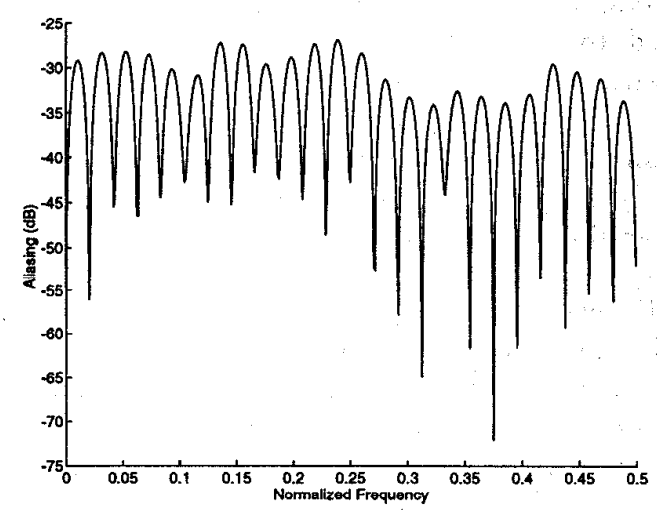

Figure 4: Global aliasing error $\left(20 \log _{10} E_{a}(F)\right)$ for the bank of Example $2(\mathrm{~N}=48)$ 